\title{
MULTI-MODALITY IMAGE REGISTRATION USING THE HOUGH TRANSFORM
}

\author{
Artur V. Cideciyan and Joachim H. Nagel \\ Department of Biomedical Engineering, University of Miami \\ PO Box 248294, Coral Gables, FL 33124-0621
}

\section{THEORY}

\section{ABSTRACT}

We present a method for finding relative translation and rotation between images of different modalities, for cases in which external contours can be approximated by ellipses. The Hough transform is used to map the feature space to the parameter space. The feature space consists of the pixel coordinates and gradient direction of all possible edge points, and the parameter space defines all acceptable ellipses. The difference between ellipse parameters, calculated separately for each image, is used to determine the relative translation and rotation. The images are assumed to be corrected for scale. The method is tested on simulated images and on pairs of actual PET/MRI images of the brain.

\section{INTRODUCTION}

Physiological images (PET, SPECT) complemented with patient specific anatomical images (CT, MRI) can be very powerful diagnostic and therapeutic tools. The use of these tools is complicated by the fact that the two images usually are not spatially matched due to the lack of exactly-reproducible patient positioning and also due to differences in scanner characteristics. Therefore considerable research has been aimed at registering physiological and anatomical information in $2 \mathrm{D}[1]$ and $3 \mathrm{D}[2,3]$.

Determination of global registration parameters using corresponding pairs of landmark points is commonly used in multimodality matching [3]. These methods rely either on application of invasive stereotactic frames or on identification of anatomical landmarks by a qualified observer. Methods which calculate global registration parameters using some kind of least-error fitting of external contour or surface points $[2,3]$, as well as the method of moment invariants [4], depend on the reliable determination of those points. These methods are usually thrown off by the presence of an abnormality causing discontinuities in the contour, such as a lesion in the PET image. Correlation method's [1] using the whole information content of the image have also been used with some success. They implicitly assume that one of the images is the noisy version of the other; an assumption not usually valid for anatomical/physiological image pairs.

In this paper, we describe a Hough Transform based matching method, which uses the magnitude and the direction of the intensity gradient to determine an ellipse corresponding to the highest number of edge points. This ellipse is assumed to represent the external contour of the image, and the differences in the ellipse parameters of both images are used to determine the relative translation and rotation. It is assumed that a global scaling factor, related to the pixel size of the scanner, is known, and that no other distortions are present. In the case of axial brain scans, the elliptic contour assumption is reasonable. The technique is especially useful when discontinuities in the contour (e.g., a lesion) exist.
We use the Hough Transform to map the feature space to the parameter space [5]. An edge operator (e.g. Sobel operator) is used in the $\mathrm{x}$ and $\mathrm{y}$ directions to generate two partial intensity gradients $\nabla_{\mathrm{x}}$ and $\nabla_{y}$ at every pixel location. The gradient magnitude and gradient direction are defined as

$$
|\nabla|=\sqrt{\nabla_{x}{ }^{2}+\nabla_{y}^{2}} \quad \phi=\arctan \left(\frac{\nabla_{y}}{\nabla_{x}}\right)
$$

An appropriate threshold is used on the gradient magnitude to determine $\mathrm{N}$ edge points with the highest gradient magnitudes. $\mathrm{N}$ is usually set to be equal to $5 \%$ of all pixels in the image. The $x, y$ coordinates of these $\mathrm{N}$ points and the direction of the gradient constitute $\mathrm{N}$ vectors in the three dimensional feature space. Note that the gradient magnitude information is discarded once the edge points are determined.

The mapping from the feature space to the parameter space for an elliptic model has been described by Ballard [6] and the same method has been applied to contour determination of nuclear medicine images of the heart by Blokland et al. [7]. A short description of this method follows:

An ellipse centered at $\left(x_{0}, y_{0}\right)$ and with major and minor diameters of $2 a$ and $2 b$, respectively, is defined as

$$
\frac{\left(x^{\prime}\right)^{2}}{a^{2}}+\frac{\left(y^{\prime}\right)^{2}}{b^{2}}=1
$$

where $\left(x^{\prime}\right)=x-x_{0}$ and $\left(y^{\prime}\right)=y-y_{0}$ are the coordinates normalized with respect to the center of the ellipse $\left(x_{0}, y_{0}\right)$. Next, (2) is differentiated (implicitly) with respect to $x$

$$
\frac{2\left(x^{\prime}\right)}{a^{2}}+\frac{2\left(y^{\prime}\right)}{b^{2}} \xi=0
$$

where $\xi=d y / d x$ is the slope of the elliptical curve. Substituting (3) into (2), and solving for $\left(x^{\prime}\right)$ and $\left(y^{\prime}\right)$, we get

$$
\left(x^{\prime}\right)= \pm \frac{a}{\sqrt{1+\frac{b^{2}}{a^{2} \xi^{2}}}} \quad\left(y^{\prime}\right)= \pm \frac{b}{\sqrt{1+\frac{a^{2} \xi^{2}}{b^{2}}}}
$$

The signs of $\left(x^{\prime}\right)$ and $\left(y^{\prime}\right)$ are determined using the signs of the partial gradients $\nabla_{x}$ and $\nabla_{y}$. If we assume a model of the image giving rise to the elliptical contour, we can relate the gradient direction $\phi$ to the slope $\xi$. In this work, a high intensity interior and a low intensity background was assumed as a model, which seems to fit both PET and MRI images of the brain. Then

$$
\xi=\tan \left(\phi-\frac{\pi}{2}-\theta\right)
$$

where $\theta$ is the rotation parameter which relaxes the previous implicit assumption that the ellipse is aligned with the axes. The calculated normalized coordinates, $\left(x^{\prime}\right)$ and $\left(y^{\prime}\right)$, are rotated by $\theta$ to compensate for rotation by $-\theta$ in (5).

Discrete values of $x_{0}, y_{0}, a, b$ and $\theta$ constitute the five dimensional parameter space sometimes called the accumulator. 
The mapping is performed as follows:

For the range of values of $a, b$ and $\theta$ and for every one of the feature vectors, the center coordinates $\left(x_{0}, y_{0}\right)$ are calculated and the nearest cell in the parameter space is incremented. Next, the maximum in the parameter space is used to define an ellipse corresponding to the highest number of edge points. If we assume that most (but not necessarily all) of the original feature vectors lie on the contour, then the calculated ellipse is representative of the external image contour.

The algorithm was written in $C$ and compiled both on an IBM/PS2 and a SUN 3/110. Due to available physical memory limitations, the implementation required a multi-resolution approach. First, a calculation with a coarse parameter space reduced the range of possible ellipses, and later a fine parameter space enabled the determination of a best fit ellipse.

\section{RESULTS}

Figure 1a shows an elliptic image $(128 \times 128,8$ bits $)$ with superimposed Gaussian noise (mean $=0$, st.dv. $=20$ ). A region on the boundary is forced to the background intensity to simulate a cold lesion. The ellipse is centered at $(63,63)$, is aligned with the coordinate axes, and has major and minor diameters of 60 and 50 , respectively. Figure $1 \mathrm{~b}$ shows the edge points determined using the Sobel operator and a $5 \%$ threshold on the gradient magnitude. Figure 10 depicts graphically the ellipse calculated using the Hough transform method: centered at $(62.6,62.0)$, rotated by -1.1 degrees and with major and minor diameters of 60.8 and 52.0 , respectively.

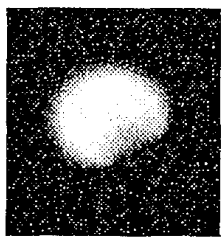

a

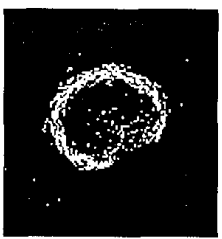

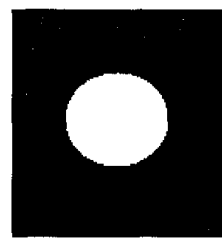

Figure 1: a) simulated image with lesion and noise, b) highest $5 \%$ gradien magnitudes of (a), c) the ellipse calculated for the edges in (b)

Figures 2a and 2d show corresponding pairs of axial PET and MRI slices of a human brain. The edge pixels of these images and the calculated ellipses can be seen in the Figures $2 b, 2 e$ and $2 c, 2 f$, respectively. The ellipse parameters show a relative translation of $(14.0,-5.0)$ and a relative rotation of 19.2 degrees.
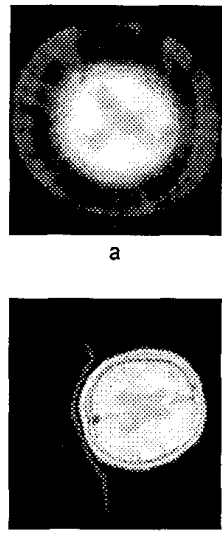
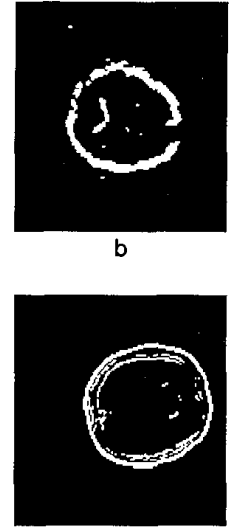

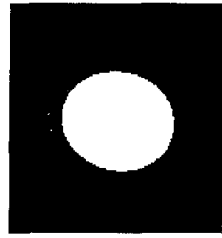

c

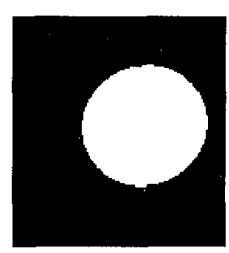

Figure 2: a,d) Representative PET and MRI images, b,e) highest $5 \%$ gradient magnitudes of (a) and (d), c,f) ellipses calculated using the edges in (b) and (e)
Figure $3 a$ was generated by superimposing of some isointensity contours of the PET image (Figure 2a) onto the MRI image (Figure 2d). The mismatch between the two images is obvious. Figure $3 \mathrm{~b}$, on the other hand, was generated by first shifting and rotating the MRI image according to the relative registration parameters calculated. As there are no universally accepted automated registration methods, it will suffice to say that the match looks reasonable to an expert observer.

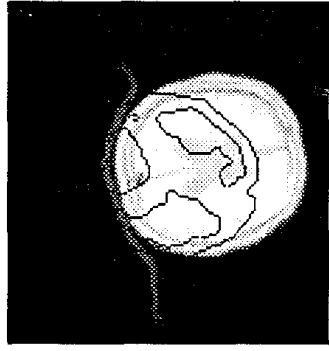

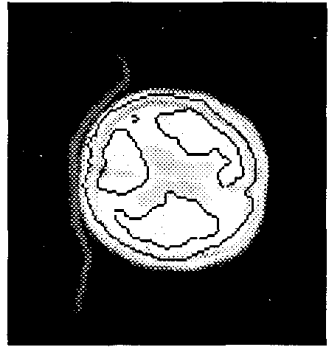

Figure 3: a) iso-intensity contours of the PET image on to the MRI image, b) same as (a), but after transformationaccording to the calculated registration parameters

\section{DISCUSSION}

We have presented a Hough transform-based method to match images of different modalities. We applied this method to a simulated image. The results show a 1 pixel error in translation and a 1 degree error in rotation. We also applied the method to a pair of axial MRI/PET brain images. The results agreed with the opinion of an expert. The assumption that the image contours can be approximated by eilipses seems to be valid in the case of axial brain images. Also, in the case of axial PET, MRI and SPECT images of the brain, a simple thresholding of the gradient magnitude will usually generate edge pixels mostly on or near the external boundary of the brain. The method has obvious limitations: a) no rotation can be determined if the image contour is circular, b) the rotations are determined modulo 180 degrees, c) scaling cannot be determined as there is no reason to believe that the edges in different modalities will correspond to the same anatomical structure, d) the method makes only limited use of the gradient magnitude information.

In conclusion, the method is most powerfut in its ability to handle the case of partial and/or noisy contours, and it can be used for matching most axial images of brain function and anatomy.

\section{Acknowledgements:}

PET and MRI images in Figures 2 and 3 were provided by the Mount Sinai Medical Center of Miami, Florida. We thank Dr. R. Duara of the Nuclear Medicine Department of the same institutionfor his expert opinion on registration quality.

\section{REFERENCES}

[1] A. Apicella, J.S. Kippenhan, J.H. Nagel, "Fast multi-modality image matching", Proc. of SPIE, 1092:252-263:, 1989.

[2] C.A. Pelizzari, G.T.Y. Chen, D.R. Spelbring, R.R. Weichselbaum, C.T. Chen, "Accurate three-dimensional registration of CT, PET, and/or MR images of the brain", J. Comp. Ass. Tom., 13:20-26, 1989.

[3] A.C. Evans, S. Marrett, L. Collins, T.M. Peters, "AnatomicalFunctional correlative analysis of the human brain using three dimensional imaging systems", Proc. of SPIE, 1092:264-274, 1989

[4] M.K. Hu, "Visual pattern recognition by moment invariants", IEEE Trans. on Information Theory, IT-8:179-187, 1962.

[5] P.V.C. Hough, "Method and means for recognizing complex patterns", US Patent 3069654: 1962.

[6] D.H. Ballard, "Generalizing the Hough transform to detect arbitrary shapes", Pattern Recognition, 13:111-122, 1981.

[7] J.A.K. Blokland, A.M. Vossepoel, A.R. Bakker, E.K.J. Pauwels, "Detection of elliptical contours", In: Mathematics and Computer Science in Medical Imaging, M.A. Viergever, A.E. Todd-Pokropek (Eds), Springer-Verlag, 1988 